\title{
Open
}

\section{High frequency of mosaic pathogenic variants in genes causing epilepsy-related neurodevelopmental disorders}

\author{
Mary Beth Stosser, MS ${ }^{1}$, Amanda S. Lindy, PhD ${ }^{1}$, Elizabeth Butler, MS ${ }^{1}$, Kyle Retterer, MS ${ }^{1}$, \\ Caitlin M. Piccirillo-Stosser ${ }^{2}$, Gabriele Richard, MD ${ }^{1}$ and Dianalee A. McKnight, PhD ${ }^{1}$
}

\begin{abstract}
Purpose: Mosaicism probably represents an underreported cause of genetic disorders due to detection challenges during routine molecular diagnostics. The purpose of this study was to evaluate the frequency of mosaicism detected by next-generation sequencing in genes associated with epilepsy-related neurodevelopmental disorders.

Methods: We conducted a retrospective analysis of 893 probands with epilepsy who had a multigene epilepsy panel or whole-exome sequencing performed in a clinical diagnostic laboratory and were positive for a pathogenic or likely pathogenic variant in one of nine genes (CDKL5, GABRA1, GABRG2, GRIN2B, KCNQ2, MECP2, $P C D H 19, S C N 1 A$, or SCN2A). Parental results were available for 395 of these probands.
\end{abstract}

Results: Mosaicism was most common in the CDKL5, PCDH19, $S C N 2 A$, and SCN1A genes. Mosaicism was observed in GABRA1,
GABRG2, and GRIN2B, which previously have not been reported to have mosaicism, and also in KCNQ2 and MECP2. Parental mosaicism was observed for pathogenic variants in multiple genes including KCNQ2, MECP2, SCN1A, and SCN2A.

Conclusion: Mosaic pathogenic variants were identified frequently in nine genes associated with various neurological conditions. Given the potential clinical ramifications, our findings suggest that next-generation sequencing diagnostic methods may be utilized when testing these genes in a diagnostic laboratory.

Genet Med advance online publication 24 August 2017

Key Words: epilepsy; genetic testing; mosaicism; next-generation sequencing; neurodevelopmental disorders

\section{INTRODUCTION}

The development and adoption of next-generation sequencing (NGS) based diagnostics have revolutionized the ability to simultaneously analyze multiple genes in an accurate and efficient manner. Compared with traditional sequencing methods, the digital nature of NGS technology allows quantification of sequence variants such that a high read depth can enable the detection of low-level mosaic variants. As diagnostic NGS is increasingly used for sequence-based variant detection, there is mounting evidence that somatic and gonosomal mosaicism are more common and play a greater role in human genetic disorders than previously recognized. In affected patients, enhanced detection of somatic mosaicism can provide a definitive molecular diagnosis to patients who may have received negative results from less sensitive testing and can also directly affect disease management. ${ }^{1}$ Additionally, the detection and reporting of mosaicism is relevant to the families of probands for accurate estimates of recurrence risk. When mosaicism is detected in apparently healthy parents of an affected child, it is essential for assigning a more accurate recurrence risk, where that risk would increase from negligible for a true de novo variant to up to the published theoretical risk of $33 \%{ }^{2}$

Mosaicism can be classified into three types depending on the affected stage of development and the affected tissue(s): germ-line mosaicism, somatic mosaicism, and gonosomal mosaicism (a combination of germ-line and somatic mosaicism). ${ }^{3}$ Owing to limitations in tissue sampling, it is difficult to precisely identify the type of mosaicism in all situations. Several genetic disorders often involve mosaicism, for example, neurofibromatosis type 1, Proteus syndrome, linear nevus sebaceous syndrome, McCune-Albright syndrome, Beckwith-Wiedemann syndrome, and tuberous sclerosis complex. Based on case reports, mosaicism also plays a role in other types of genetic disorders such as neurodevelopmental and epilepsy-related disorders. ${ }^{4-11}$

To date, the frequency of mosaicism in neurodevelopmental disorders has been evaluated mostly in research studies of individuals with disorders such as autism and intellectual disability. ${ }^{12,13}$ A systematic study of the role of mosaicism in epilepsy or other neurodevelopmental disorders, especially in a clinical diagnostic setting, is currently lacking. Although it has been hypothesized that the frequency of mosaicism in certain epilepsy-related genes may be higher than previously appreciated, no information is available regarding the overall frequency of mosaic pathogenic variants in specific genes or disorders associated with epilepsy. ${ }^{4-11}$ Additionally, parental mosaicism has been reported in some epilepsy-related genes. For example, a high incidence (at least $7 \%$ ) of parental mosaicism has been detected by direct sequencing in families with inherited SCN1A-related epilepsies. In these families, the parents included individuals who were asymptomatic, mildly 


\section{ORIGINAL RESEARCH ARTICLE}

affected, and severely affected, though this type of clinical information for other genes may not be available. ${ }^{14}$

In this study, we report for the first time the extent and level of mosaicism detectable by multigene or whole-exome NGS for patients with epilepsy undergoing testing in a clinical diagnostic laboratory.

\section{Patient population}

MATERIALS AND METHODS

The cohort included 893 probands, referred from December 2011 to December 2015, who had undergone NGS testing for either a panel of up to 70 epilepsy-related genes or wholeexome sequencing (WES) and were found to be positive for a pathogenic or likely pathogenic variant in one of the following genes: CDKL5, GABRA1, GABRG2, GRIN2B, KCNQ2, MECP2, PCDH19, SCN1A, or SCN2A (Figure 1). These nine genes were selected because mosaic pathogenic and likely pathogenic variants were observed in one or more probands with epilepsy by NGS and frequencies of mosaicism for these genes have not been previously reported, to our knowledge. In addition to epilepsy, clinical indications for testing often included other neurodevelopmental features such as developmental delay, intellectual disability, learning disability, developmental regression, autism spectrum disorder, behavioral/psychiatric abnormalities, hypotonia, microcephaly/macrocephaly, and movement disorders. Clinical information, family history, and ethnicity were provided using a clinical checklist and/or clinic notes submitted by the referring clinicians, who were primarily neurologists or geneticists working in private practice, private hospitals, or university-based medical centers. The majority of patients resided in the United States or Canada. In a subset of probands with heterozygous or hemizygous pathogenic or likely pathogenic variants, parental mosaicism was evaluated either by NGS or targeted Sanger sequencing. Informed consent for clinical testing was obtained by the referring clinicians.

\section{Next-generation sequencing}

A targeted capture panel (ranging from 18 to 70 genes) or WES was used to investigate genes associated with epilepsyrelated disorders. For all panels, targeted regions were enriched from genomic DNA extracted from blood using either multiplex polymerase chain reaction (PCR) by Raindance Technologies (Raindance Technologies, Lexington, MA) or RNA base hybridization capture using a custom designed library and SureSelect kit (Agilent Technologies, Santa Clara, CA) following the manufacturer's instructions. Specifically, for libraries generated with Raindance Technologies (Billerica, MA), DNA was sheared on a Covaris instrument (Woburn, MA) to produce 5-kb fragments that were purified using the Qiagen QIAQuick (Hilden, Germany) mini-elute kit. DNA fragments were merged with PCR primers in microdroplets and transferred to PCR tubes for PCR amplification. The amplified products were enzymatically fragmented and tagged with adaptors using the standard
STOSSER et al | Mosaic variants in genes associated with epilepsy-related NDD
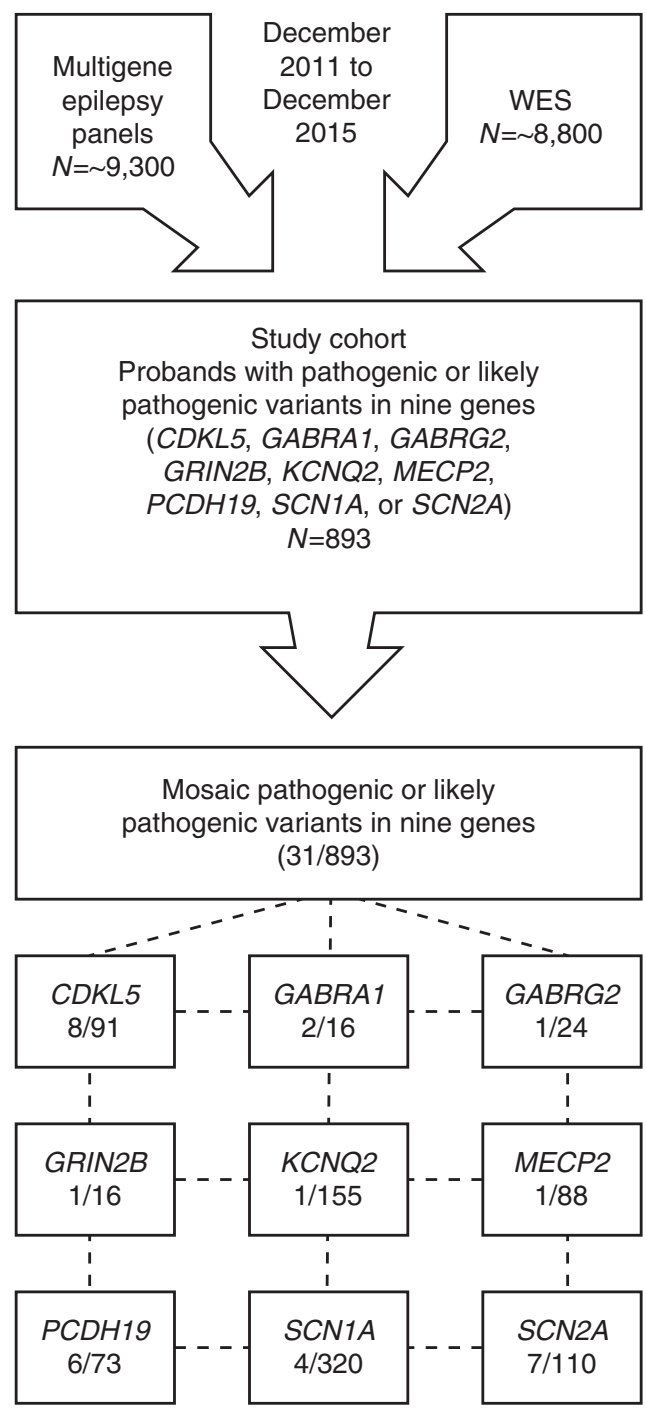

Figure 1 Flow chart of testing. Schematic overview of the breakdown of testing for the study cohort. WES, whole-exome sequencing.

Nextera protocol (Illumina, San Diego, CA). A final PCR enrichment amplification was performed at $65^{\circ} \mathrm{C}$ for 15 cycles. Products were purified using the Qiagen QIAQuick mini-elute kit. For Agilent capture processing, DNA was sheared into approximately 400-bp fragments, which were then repaired, ligated to adaptors, and purified for subsequent PCR amplification. Amplified products were then captured by biotinylated RNA library baits in solution following the manufacturer's instructions. Bound DNA was isolated with streptavidin-coated beads and reamplified.

The final library products for both methods were sequenced using the Illumina HiSeq 2000 or 2500 sequencing system with either 50-bp single-end or 100-bp paired-end reads, respectively (Illumina, San Diego, CA), following manufacturer's instructions and described previously. ${ }^{15}$ All targeted gene regions were created as custom contigs based on RefSeq gene sequences. For Raindance, reads were mapped to only the custom contig targets, while for capture, to avoid 
misalignment of any genomic carryover, read alignment used the curated gene regions plus a masked version of hg19 to filter any genomic carryover. Local realignment around insertion/deletion sites was performed using the Genome Analysis Toolkit v1.6. ${ }^{16}$ Regions of interest were defined as the exon and flanking noncoding sequences. All samples were sequenced to an average coverage of at least $1200 \times$. Positions within the region of interest covered at less than $15 \times$ were compensated for by conventional Sanger DNA sequencing using an ABI 3730 (Life Technologies, Carlsbad, CA) and standard protocols. All variants called within the region of interest by any of these programs were analyzed, and all novel variants that appeared real based on manual inspection of NGS alignments and all previously classified, nonbenign variants were confirmed by conventional Sanger DNA sequence analysis with a new DNA preparation.

For WES, genomic DNA was extracted from whole blood of affected probands and, when available, their parents and other similarly affected relatives. Next-generation sequencing was performed on exon targets captured using the Agilent SureSelect Human All Exon V4 (SS V4) or Clinical Research Exome kit (Agilent Technologies) to an average target depth of more than $100 \times$. The sequencing methodology, data processing pipeline, and variant filtering and interpretation protocol have been described previously. ${ }^{17}$ All clinically relevant variants were confirmed by conventional Sanger DNA sequence analysis with a new DNA preparation.

The read depth varied based on the specific NGS method, Raindance, custom capture, or whole exome sequencing (Supplementary Table S1 online and Supplementary Figure S1). Supplementary Figure S1 shows that the pertarget coverage is more consistent for capture-based methods. The capture panel provides the best coverage for consistent mosaic variant calling with nearly all targets consistently covered to $>100 \times$ and most targets consistently covered to $>200 \times$. While the Raindance panel provides higher coverage for some targets, its coverage uniformity is poor.

\section{Variant analysis and interpretation}

Mosaicism was hypothesized when variants in autosomal genes and X-linked genes in females were observed at a lowerthan-expected ratio of variant to wild-type NGS reads and exhibited unequal amplification by Sanger sequencing. For males, mosaicism was suspected for X-linked genes when both variant and wild-type alleles were observed and when there was no indication of a sex chromosome aneuploidy based on either concurrent exon-level array comparative genomic hybridization data or prior chromosome analysis by karyotype.

The general assertion criteria for variant classification are publicly available on the GeneDx ClinVar submission page (http://www.ncbi.nlm.nih.gov/clinvar/submitters/26957/). Variants were categorized using the five-tier classification system recommended by the American College of Medical Genetics and Genomics. Clinical variant interpretation was conducted in the context of the proband's phenotype when available. Likely pathogenic variants have a high probability of being pathogenic. Pathogenic and likely pathogenic variants were used for the analysis of this manuscript. For the sake of simplicity, all of the qualifying variants are referred to as "pathogenic" in this manuscript, although many are classified in ClinVar as "likely pathogenic."

\section{Mosaicism in probands}

\section{RESULTS}

Molecular diagnostic testing for epilepsy-related disorders utilizing NGS yielded positive diagnostic results in 893 probands, including pathogenic variants in nine genes analyzed (CDKL5, GABRA1, GABRG2, GRIN2B, KCNQ2, $M E C P 2, P C D H 19, S C N 1 A$, and SCN2A) (Figure 1). For the multigene NGS panels, more than 9,300 probands were tested in the specified time period, and these nine genes accounted for approximately $57 \%$ of the overall positive cases. Among all positive cases identified through the multigene NGS panels, missense variants accounted for the majority (52\%) of pathogenic variants detected; frameshift accounted for $23 \%$, nonsense $14 \%$, splice $10 \%$, and insertion/deletion 1\%. Among probands with positive results in these nine genes $(n=893$, Supplementary Figure S1), 31 demonstrated a lower-thanexpected ratio of variant to wild-type NGS reads, which is indicative of mosaicism in the nine selected genes. The mean read depth at the site of pathogenic changes was 1,299 (range: 42-3,444 reads) (Table 1). The level of observed mosaicism ranged from 9 to $40 \%$ of sequencing reads in probands with autosomal dominant disorders and females with X-linked disorders. Males with an X-linked disorder had mosaic variants in $8-87 \%$ of sequencing reads (Table 1 ). In this study, the breakdown of the types of mosaic variants in these nine genes were missense $(68 \%)$, nonsense $(16 \%)$, frameshift (10\%), and splice site (6\%) variants (Table 1). The mean read depth across the nine genes with mosaic pathogenic variants is provided in Supplementary Table S1 for each of the different testing methods. Density plots of per-sample, per-target coverage for each of the four methods are illustrated in Supplementary Figure S1.

The highest frequency of mosaicism among positive probands was observed for two X-linked genes, $8.8 \%$ for CDKL5 (0.088; 95\% CI, 0.039-0.166) and 8.2\% for PCDH19 (0.082; 95\% CI, 0.031-0.170) (Table 2). Of the individuals with pathogenic variants in CDKL5, 21\% (19/91) were male. Additionally, $75 \%(6 / 8)$ of mosaic pathogenic variants identified in CDKL5 were in affected males noted to have epilepsy and/or developmental delay, and 25\% (2/8) were in affected females with epilepsy and developmental delay. Although PCDH19-related epilepsy is an X-linked dominant disorder almost exclusively affecting females, five males were found to be mosaic for a pathogenic variant in the PCDH19 gene and presented with epilepsy and/or developmental delay, similar to females with $\mathrm{PCDH} 19$ pathogenic variants.

Mosaic pathogenic variants were observed in $6.4 \%$ of patients with pathogenic variants in SCN2A $(0.064 ; 95 \% \mathrm{CI}$, 0.026-0.128) (Table 2). All patients with mosaic SCN2A 
\&̊ Table 1 Cases with mosaic pathogenic variant(s)

Gene Gender Variant (hg19 position; coding DNA; protein)

Type of variant

Autosomal dominant inheritance

GABRA1 Male chr5:161317988 T>C; c.788 T>C; p.Met263Thr

GABRA1 Male chr5:161317999 C > A; c.799 C > A; p.Leu267lle

GABRG2 Male chr5:161522522 C>A; c.281 C>A; p.Thr94Lys

GRIN2B Male chr12:13720105 A > C; c.2452 A > C; p.Met818Leu

KCNQ2 Female chr20:62044866 T>A; c.1700 T>A; p.Val567Asp

SCN1A Male chr2:166852531 C > T; c.4573 C > T; p.Arg1525Ter

SCN1A Male chr2:166915131 T>A; c.332 T>A; p.Leu111Ter

SCN1A Female chr2:166903453 T>C; c.1204 T>C; p.Phe402Leu

SCN1A Female chr2:166929992 A >-; c.140delA; p.Asn47MetfsTer45

SCN2A Male chr2:166201203 C > G; c.2701 C > G; p.Gln901Glu

SCN2A Male chr2:166198881 G>A; c.2464 G>A; p.Gly822Ser

SCN2A Female chr2:166237619 T>A; c.4463 T>A; p.lle1488Asn

SCN2A Female chr2:166201153 T>A; c.2651 T>A; p.Leu884His

SCN2A Female chr2:166165888 G>A; c.632 G>A; p.Gly211Asp

SCN2A $A^{\text {a }}$ Male chr2:166231223 T>C, T>A

c.4001 T>C, c.4001 T>A; p.lle1334Thr, p.lle1334Asn
SCN2A Male chr2:166210777 G > A; c.2995 G > A; p.Glu999Lys

Testing

method

$\%$ of variant reads

$X$-linked inheritance

CDKL5 Fema

CDKL5 Male

CDKL5 Female

CDKL5 Male

CDKL5 Male

CDKL5 Male

CDKL5 Male

CDKL5 Male

MECP2 Male

PCDH19 Male

PCDH19 Female

PCDH19 Male

PCDH19 Male

PCDH19 Male

chrX:18613489 C > T; c.766 C > T; p.Gln256Ter

chrX:18602452 G>A; c.533 G > A; p.Arg178Gln

chrX:18600056 A>G; c.449 A>G; p.Lys150Arg

chrX:18627690 G>A; c.2152 G > A; p.Val718Met

chrX:18598085 C> T; c.400 C > T; p.Arg134Ter

chrX:18622719 C > T; c.1675 C > T; p.Arg559Ter

chrX:18528948 C > A; c.73 G > A; p.Gly25Arg

chrX:18627686-18627687 CA > -; c.2148_2149delCA; p.Tyr716Ter

chrX:153297671 G>A; c.364 G>A; p.Val122Met

chrX:99661637-99661640 CTCT> -; c.1956_1959delCTCT; p.Ser653ProfsTer6

chrX:99662962 G > C; c.634 G > C; p.Asp212His

chrX:99605642 T>C; C.2534+2 T>C

chrX:99661447 T>C; c.2147+2 T>C

chrX:99661723 A> G; c.1873 A>G; p.Arg625Gly

chrX:99663226 G>C; c.370 G > C; p.Asp124His

ICA; p.Tyr716Ter

PCDH19 Male
ing.

aSingle patient is mosaic for two different nucleotide substitutions at same codon, known as double mosaicism.

$\begin{array}{llrr} & & & \\ \text { Missense } & \text { Raindance } & 11.7 \% & 803 \\ \text { Missense } & \text { Raindance } & 18.6 \% & 483 \\ \text { Missense } & \text { Raindance } & 14.4 \% & 667 \\ \text { Missense } & \text { Custom capture } & 24.4 \% & 2,574 \\ \text { Missense } & \text { Custom capture } & 30.2 \% & 463 \\ \text { Nonsense } & \text { Raindance } & 9.2 \% & 1,021 \\ \text { Nonsense } & \text { Custom capture } & 20.2 \% & 3,406 \\ \text { Missense } & \text { Custom capture } & 24.7 \% & 3,107 \\ \text { Frameshift } & \text { Raindance } & 26.0 \% & 412 \\ \text { Missense } & \text { Raindance } & 11.6 \% & 627 \\ \text { Missense } & \text { Custom capture } & 13.0 \% & 3,444 \\ \text { Missense } & \text { WES } & 14.7 \% & 95 \\ \text { Missense } & \text { Raindance } & 16.8 \% & 1,413 \\ \text { Missense } & \text { Raindance } & 20.3 \% & 488 \\ \text { Missense } & \text { Custom capture } & 22.1 \% & 961 \\ \text { Two missense at same } & \text { Custom capture } & 27.1 \% & 3,320 \\ \text { nucleotide position } & & 39.5 \% & 3,320\end{array}$

\begin{tabular}{llrr} 
& & & \\
Nonsense & Custom capture & $10.1 \%$ & 2,353 \\
Missense & WES & $14.3 \%$ & 42 \\
Missense & Raindance & $16.0 \%$ & 562 \\
Missense & Raindance & $25.3 \%$ & 2,961 \\
Nonsense & Raindance & $31.1 \%$ & 380 \\
Nonsense & WES & $36.1 \%$ & 180 \\
Missense & Raindance & $41.2 \%$ & 284 \\
Frameshift & Raindance & $56.3 \%$ & 895 \\
Missense & Custom capture & $31.4 \%$ & 1,797 \\
Frameshift & Custom capture & $8.1 \%$ & 1,551 \\
Missense & Raindance & $12.5 \%$ & 2,556 \\
Splice & Raindance & $22.2 \%$ & 189 \\
Splice & Custom capture & $43.6 \%$ & 1,338 \\
Missense & Raindance & $83.1 \%$ & 688 \\
Missense & Raindance & $87.2 \%$ & 1,219 \\
\hline & & &
\end{tabular}




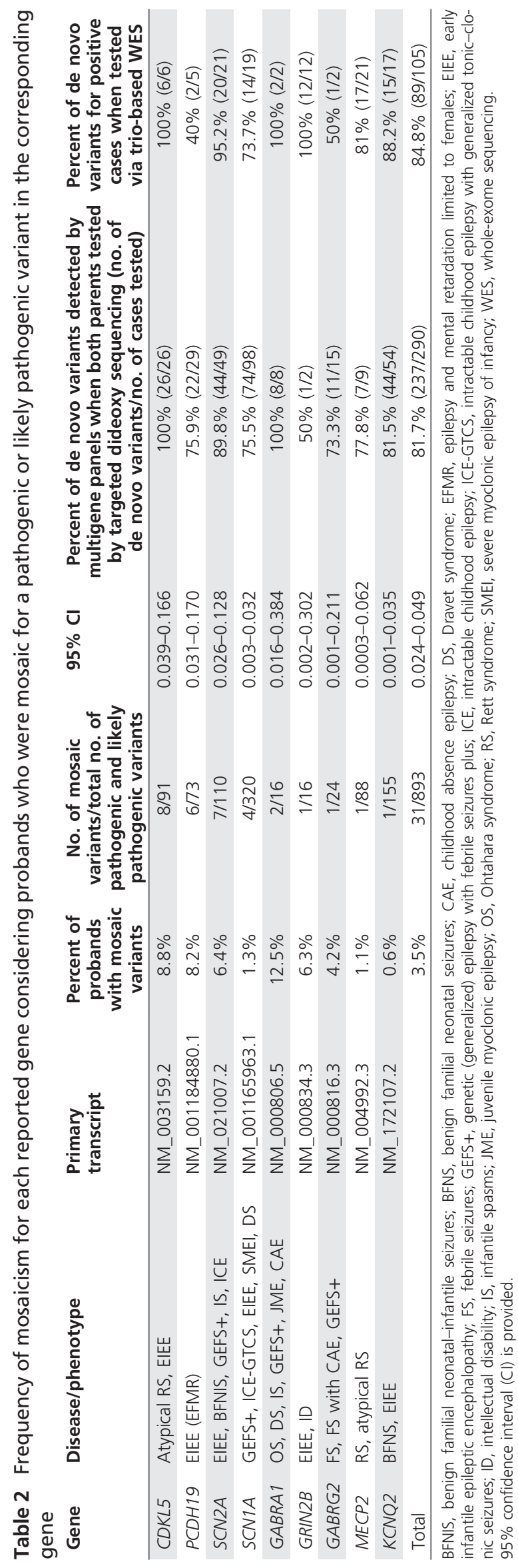

pathogenic variants had epilepsy and many (5/7) were also noted to have developmental delay based on limited clinical information provided. Additionally, an unusual finding of double mosaicism, previously referred to as triple mosaicism, ${ }^{18}$ was identified in one patient who was mosaic for two different pathogenic variants at the same nucleotide position in the SCN2A gene, with each sequence change having different variant allele frequencies (Table 1). The frequency of mosaicism in SCN1A $(0.013$; 95\% CI, 0.003$0.032)$ was significantly lower than in $S C N 2 A(P<0.01$; twotailed Fisher's exact test) (Table 2).

Of note, we also detected mosaic pathogenic variants in several additional epilepsy-related genes including some of the GABA receptor genes, GABRA1 (0.125; 95\% CI, 0.016-0.384) and GABRG2 (0.042; 95\% CI, 0.001-0.211); GRIN2B (0.063; 95\% CI, 0.002-0.302), which encodes the glutamate-binding NR2B subunit of the $\mathrm{N}$-methyl-D-aspartate receptor; the potassium channel gene KCNQ2 (0.006; 95\% CI, 0.001$0.035)$; and MECP2 $(0.011 ; 95 \% \mathrm{CI}, 0.0003-0.062)$ in a male with atypical Rett syndrome (Table 2).

These data indicate that the overall frequency of mosaic pathogenic variants in the two genes, CDKL5 and PCDH19 (0.085; 95\% CI, 0.048-0.139), was significantly higher than the overall frequency of mosaic pathogenic variants in the other seven genes combined, GABRA1, GABRG2, GRIN2B, KCNQ2, MECP2, SCN1A, and SCN2A (0.023; 95\% CI, 0.0140.037), $(P<0.01$; two-tailed Fisher's exact test $)$.

\section{Mosaicism in parents}

Parental testing was performed for probands undergoing triobased WES and recommended for all probands with positive results identified via panel testing. For probands tested by multigene NGS panels and identified to have pathogenic variants in CDKL5, GABRA1, GABRG2, GRIN2B, KCNQ2, MECP2, PCDH19, SCN1A, or SCN2A, 290 sets of parents underwent targeted testing for the variant by Sanger sequencing. Of these, $237(81.7 \%)$ were determined to be de novo and 53 (18.3\%) were inherited (Table 2). Similarly, for probands tested by WES trio with a pathogenic variant in one of these nine genes, the overall frequency of de novo variants was $84.8 \%$ (89/105) (Table 2). For parents tested either by targeted Sanger sequencing after the proband had a positive result by multigene NGS panel or by WES trio testing, we observed that 12 parents were suspected to have mosaic variants in either SCN1A (6), SCN2A (3), KCNQ2 (2), or MECP2 (1) (Table 3). Clinical information was not provided for one parent, but it was provided for the other 11 parents. Based on the clinical information provided, 27\% (3/11) of these parents were clinically affected with clinical features consistent with the disorders associated with these genes (Table 3). However, two of the three affected parents were reported to have milder or later-onset symptoms compared with their children. The remaining 73\% (8/11) of the parents who carried a mosaic pathogenic variant and for whom clinical information was provided were reportedly unaffected (Table 3). In addition, we observed one case of suspected 


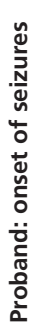

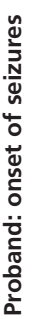
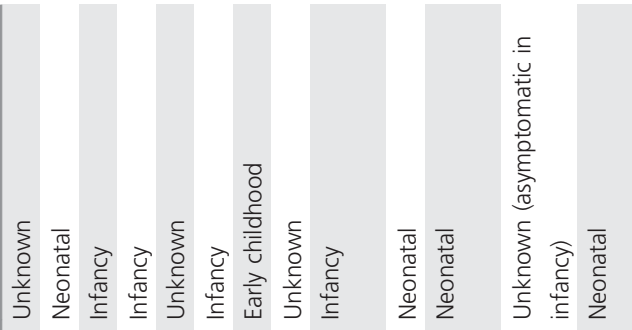

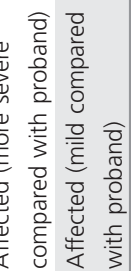
early infantile epileptic encephalopathy that is sometimes referred to as epilepsy and mental retardation limited to females. This condition follows an unusual X-linked inheritance pattern in which females are affected while males who are hemizygous for a pathogenic variant are unaffected carriers. Although the mechanism of disease is not completely understood, cellular interference has been speculated.,24 Mosaic pathogenic variants have been reported in affected males and females. ${ }^{7,8,11,24-26}$ We identified mosaic pathogenic variants in $P C D H 19$ in over $8 \%$ of patients with a $P C D H 19-$ related disorder. As expected based on the hypothesized dominant-negative etiology for PCDH19-related disorders, all males with a mosaic pathogenic $P C D H 19$ variant were found to be clinically affected. Additionally, two affected males in our cohort were hemizygous for pathogenic variants in the PCDH19 gene (one frameshift and one missense); these patients had no evidence of wild-type allele in the DNA sample extracted from blood. It is possible that the phenotype in these male patients was not caused by the pathogenic PCDH19 variants identified and the patients were coincidentally found to be carriers of the variants. Another possibility is that these male patients are mosaic, but we were unable to detect the wild-type allele in the provided samples extracted 
from blood. A prior publication included a report of a male who appeared hemizygous for a pathogenic PCDH19 variant with no evidence of mosaicism in one tissue (blood), while a sample from another tissue (skin) revealed the presence of mosaicism. ${ }^{7}$ In the patients reported here, alternate tissue types were not submitted, limiting certain conclusions.

The SCN2A gene encodes the alpha subunit of a neuronal sodium channel, which mediates normal neuronal firing. ${ }^{27}$ Pathogenic variants in SCN2A cause benign familial neonatalinfantile seizures, neonatal epileptic encephalopathy, and other types of epilepsy. ${ }^{27,28}$ Mosaic pathogenic variants in $S C N 2 A$ have only been reported in the literature in two cases to date. ${ }^{29,30}$ Paternal germ-line mosaicism for an SCN2A pathogenic variant that was transmitted to two affected halfsiblings has also been reported. ${ }^{31}$ Our data demonstrate that more than $6 \%$ of patients with a pathogenic SCN2A variant were mosaic.

The SCN1A gene encodes the alpha subunit of a neuronal voltage-gated sodium channel that regulates the excitability of neurons. ${ }^{32}$ Pathogenic variants in SCN1A cause a variety of epilepsy phenotypes, ranging from simple febrile seizures to severe infantile epileptic encephalopathies. The frequency of mosaic pathogenic variants detected in SCN1A in probands was $1.3 \%$; the overall frequency of mosaicism in SCN1A that we observed for families with pathogenic variants in the SCN1A gene was $3.1 \%(10 / 320)$. In addition to the four probands found to harbor mosaic pathogenic variants in $S C N 1 A$, there were six instances of parental mosaicism for SCN1A pathogenic variants. The majority of parents with mosaic SCN1A pathogenic variants were reportedly unaffected. These data support previous reports that parental mosaicism in SCN1A may be more common than previously thought. ${ }^{33}$ In addition, other investigators have indicated that parental mosaicism was not detected in their testing cohort by targeted Sanger sequencing, and that more sensitive tools for detecting low-level mosaicism in parents could allow for more accurate diagnosis. ${ }^{33-35}$

Mosaic pathogenic variants were also observed in the GABRA1, GRIN2B, GABRG2, MECP2, and KCNQ2 genes. Although previous case studies have included reports of mosaic pathogenic variants in KCNQ2 and MECP2 in probands and/or their parents, ours is, to our knowledge, the first report of mosaic variants in the GABRA1, GABRG2, and GRIN2B genes. Although these genes had a relatively high frequency of mosaicism, limited data were available due to the small number of positive cases with pathogenic variants in these genes (Table 2).

We report parental mosaicism for pathogenic variants in the SCN1A, SCN2A, KCNQ2, and MECP2 genes. Parental mosaicism in these genes has been reported in unaffected, mildly affected, and more severely affected parents; therefore, the likelihood of parental mosaicism appears to be difficult to predict based on parental phenotype..$^{5,34,36-39}$ In our data set, of the parents who were identified as mosaic carriers of a pathogenic variant, and for whom we received clinical information, $73 \%(8 / 11)$ were reported to have no related neurological features. The parents identified as mosaic carriers of a pathogenic variant who were also reported to have a neurodevelopmental phenotype typically had features that were less severe than the features reported for their affected child. Taken together, our NGS-based data suggest that parental mosaicism may be a relatively frequent and underappreciated occurrence for pathogenic variants in genes causing epilepsy, and that the possibility of parental mosaicism should be considered when providing counseling about reproductive risks to parents of a child with a pathogenic variant in an epilepsy-related gene. Targeted parental testing is routinely performed in clinical laboratories utilizing Sanger sequencing, which is a qualitative assay and therefore has limited sensitivity in detecting mosaicism. NGS with high read depth should be considered for parental samples when testing for variants in genes with a high frequency of mosaic pathogenic variants for better sensitivity of low-level mosaicism.

In summary, individuals with epilepsy who previously tested negative for pathogenic variants in these genes by Sanger sequencing may benefit from a repeat analysis using NGS, which is more sensitive in the detection of mosaic variants. For any proband with a pathogenic variant in these genes, targeted testing of both parents is indicated and should be performed by NGS or another quantitative assay to better evaluate for possible parental mosaicism and more accurately estimate the recurrence risk. A careful and systematic review of laboratory diagnostic data may reveal that the frequency of mosaicism in genes associated with other disorders is higher than currently reported. To determine this, the methodology and threshold of variant calls would need to be optimized for consistent detection of low-level mosaicism and multiple tissue types would need to be screened. This endeavor could possibly be accomplished by multicenter collaborations and/ or clinic-research collaborations.

\section{SUPPLEMENTARY MATERIAL}

Supplementary material is linked to the online version of the paper at http://www.nature.com/gim

\section{ACKNOWLEDGMENTS}

We gratefully acknowledge all the past and present members of the neurogenetics, laboratory, and bioinformatics teams at GeneDx.

\section{DISCLOSURE}

This cohort was tested at GeneDx and reviewed by M.B.S., A.S.L., E.B., K.R., G.R., and D.A.M., all of whom are employees of GeneDx, a wholly owned subsidiary of OPKO Health.

\section{REFERENCES}

1. Qin L, Wang J, Tian X, et al. Detection and quantification of mosaic mutations in disease genes by next-generation sequencing. J Mol Diagn 2016;18:446-453.

2. Campbell M, Stewart JR, James RA, et al. Parent of origin, mosaicism, and recurrence risk: probabilistic modeling explains the broken symmetry of transmission genetics. Am J Hum Genet 2014;95:345-359. 
3. Biesecker LG, Spinner NB. A genomic view of mosaicism and human disease. Nat Rev Genet 2013;14:307-320.

4. Bartnik M, Derwińska K, Gos M, et al. Early-onset seizures due to mosaic exonic deletions of CDKL5 in a male and two females. Genet Med 2011;13:447-452.

5. Christodoulou J, Ho G. MECP2-related disorders. In: Pagon RA, Adam MP, Ardinger $\mathrm{HH}$, et al. (eds). GeneReviews. University of Washington: Seattle, WA, 28 June 2012. https://www.ncbi.nlm.nih.gov/books/NBK1497/. 16 February, 2016.

6. Clayton-Smith J, Watson P, Ramsden S, Black GC. Somatic mutation in $M E C P 2$ as a non-fatal neurodevelopmental disorder in males. Lancet 2000;356:830-832.

7. Depienne C, Bouteiller D, Keren B, et al. Sporadic infantile epileptic encephalopathy caused by mutations in $P C D H 19$ resembles Dravet syndrome but mainly affects females. PLoS Genet 2009;5:e1000381.

8. Dibbens LM, Kneen R, Bayly MA, et al. Recurrence risk of epilepsy and mental retardation in females due to parental mosaicism of PCDH19 mutations. Neurology 2011;76:1514-1519.

9. Masliah-Plachon J, Auvin S, Nectoux J, Fichou Y, Chelly J, Bienvenu T Somatic mosaicism for a CDKL5 mutation as an epileptic encephalopathy in males. Am J Med Genet 2010;152A:2110-2111.

10. Mei D, Darra F, Barba $C$, et al. Optimizing the molecular diagnosis of CDKL5 gene-related epileptic encephalopathy in boys. Epilepsia 2014;55: 1748-1753.

11. Terracciano A, Trivisano M, Cusmai R, et al. $P C D H 19-$ related epilepsy in two mosaic male patients. Epilepsia 2016;57:e51-55.

12. Freed $D$, Pevsner J. The contribution of mosaic variants to autism spectrum disorder. PLoS Genet 2016;12:e1006245.

13. Acuna-Hidalgo R, Bo T, Kwint MP, et al. Post-zygotic point mutations are an underrecognized source of de novo genomic variation. Am J Hum Genet 2015;97:67-74.

14. Depienne C, Trouillard O, Gourfinkel-An I, et al. Mechanisms for variable expressivity of inherited SCN1A mutations causing Dravet syndrome. $J$ Med Genet 2010;47:404-410.

15. Susswein LR, Marshall ML, Nusbaum R, et al. Pathogenic and likely pathogenic variant prevalence among the first 10,000 patients referred for next-generation cancer panel testing. Genet Med 2016;18:823-832.

16. DePristo MA, Banks E, Poplin R, et al. A framework for variation discovery and genotyping using next-generation DNA sequencing data. Nat Genet 2011;43:491-498.

17. Retterer K, Juusola J, Cho MT, et al. Clinical application of whole-exome sequencing across clinical indications. Genet Med 2016;18:696-704.

18. Draaken $M$, Giesen $C A$, Kesselheim $A L$, et al. Maternal de novo triple mosaicism for two single OCRL nucleotide substitutions (c.1736A $>$ T, c. 1736A $>$ G) in a Lowe syndrome family. Hum Genet 2011;129: 513-519.

19. Artuso R, Mencarelli MA, Polli R, et al. Early-onset seizure variant of Rett syndrome: definition of the clinical diagnostic criteria. Brain Dev 2010;32: $17-24$.

20. Russo S, Marchi M, Cogliati F, et al. Novel mutations in the CDKL5 gene, predicted effects and associated phenotypes. Neurogenetics 2009;10: 241-250.

21. Bahi-Buisson N, Nectoux J, Rosas-Vargas $\mathrm{H}$, et al. Key clinical features to identify girls with CDKL5 mutations. Brain 2008;131:2647-2661.

22. Boutry-Kryza N, Ville D, Labalme $\mathrm{A}$, et al. Complex mosaic CDKL5 deletion with two distinct mutant alleles in a 4-year-old girl. Am J Med Genet 2014;164A:2025-2028.

23. Kato $T$, Morisada $\mathrm{N}$, Nagase $\mathrm{H}$, et al. Somatic mosaicism of a CDKL5 mutation identified by next-generation sequencing. Brain Dev 2015;37: 911-915.

24. Depienne C, LeGuern E. PCDH19-related infantile epileptic encephalopathy: an unusual X-linked inheritance disorder. Hum Mutat 2012;33: 627-634.
25. Terracciano A, Specchio N, Darra F, et al. Somatic mosaicism of $P C D H 19$ mutation in a family with low-penetrance EFMR. Neurogenetics 2012;13: 341-345.

26. Thiffault I, Farrow E, Smith L, et al. PCDH19-related epileptic encephalopathy in a male mosaic for a truncating variant. Am J Med Genet 2016;170:1585-1589.

27. Shi $X$, Yasumoto $S$, Kurahashi $H$, et al. Clinical spectrum of SCN2A mutations. Brain Dev 2012;34:541-545.

28. Howell KB, MCMahon JM, Carvill GL, et al. SCN2A encephalopathy: a major cause of epilepsy of infancy with migrating focal seizures. Neurology 2015;85:958-966.

29. Nakamura $\mathrm{K}$, Kato $\mathrm{M}$, Osaka $\mathrm{H}$, et al. Clinical spectrum of SCN2A mutations expanding to Ohtahara syndrome. Neurology 2013;81: 992-998.

30. Thiels C, Hoffjan S, Köhler C, Wolff M, Lücke T. SCN2A a sequence variant in mosaic state in a patient with infantile epileptic encephalopathy. Neuropediatrics 2016;47:PS01-PS11.

31. Zerem A, Lev D, Goldberg-Stern $H$, et al. Paternal germline mosaicism of a SCN2A mutation results in Ohtahara syndrome in half siblings. Eur $J$ Paediatr Neurol 2014;18:567-571.

32. Miller IO, Sotero de Menezes MA. SCN1A-related seizure disorders, In: Pagon RA, Adam MP, Ardinger HH, et al. (eds). GeneReviews. University of Washington: Seattle, WA, 15 May 2014. https://www.ncbi.nlm.nih. gov/books/NBK1318/. 16 February, 2016

33. $\mathrm{Xu} X, \mathrm{Yang} \mathrm{X}, \mathrm{Wu} \mathrm{Q}$, et al. Amplicon resequencing identified parental mosaicism for approximately $10 \%$ of "de novo" SCN1A mutations in children with Dravet syndrome. Hum Mutat 2015;36:861-872.

34. Depienne C, Arzimanoglou A, Trouillard O, et al. Parental mosaicism can cause recurrent transmission of SCN1A mutations associated with severe myoclonic epilepsy of infancy. Hum Mutat 2006;27:389.

35. Campbell IM, Yuan B, Robberecht $C$, et al. Parental somatic mosaicism is underrecognized and influences recurrence risk of genomic disorders. Am J Hum Genet 2014;95:173-182.

36. Kato M, Yamagata T, Kubota M, et al. Clinical spectrum of early onset epileptic encephalopathies caused by KCNQ2 mutation. Epilepsia 2013:54:1282-1287.

37. Marini C, Mei D, Helen Cross J, Guerrini R. Mosaic SCN1A mutation in familial severe myoclonic epilepsy of infancy. Epilepsia 2006;47: 1737-1740.

38. Miceli F, Soldovieri MV, Joshi N, Weckhuysen S, Cooper E, Taglialatela M. KCNQ2-related disorders. In: Pagon RA, Adam MP, Ardinger $\mathrm{HH}$, et al. (eds). GeneReviews. University of Washington: Seattle, WA, 31 March 2016. https://www.ncbi.nlm.nih.gov/books/NBK32534/. 16 February, 2016.

39. Milh $M$, Lacoste $C$, Cacciagli $P$, et al. Variable clinical expression in patients with mosaicism for KCNQ2 mutations. Am J Med Genet 2015;167A:2314-2318

This work is licensed under a Creative Commons Attribution 4.0 International License. The images or other third party material in this article are included in the article's Creative Commons license, unless indicated otherwise in the credit line; if the material is not included under the Creative Commons license, users will need to obtain permission from the license holder to reproduce the material. To view a copy of this license, visit http://creativecommons.org/licenses/by/4.0/

(c) The Author(s) 2018 